13

\title{
Адсорбция атомов бария на карбиде кремния
}

\author{
() С.Ю. Давыдов ${ }^{1}$, О.В. Посредник ${ }^{2}$ \\ ${ }^{1}$ Физико-технический институт им. А.Ф. Иофрфе РАН, Санкт-Петербург, Россия \\ ${ }^{2}$ Санкт-Петербургский государственный электротехнический университет „ЛЭТИ“, Санкт-Петербург, Россия \\ E-mail: Sergei_Davydov@mail.ru
}

Поступило в Редакцию 24 сентября 2019г.

В окончательной редакции 24 сентября 2019 r.

Принято к публикации 1 октября 2019г.

Адсорбция атомов бария на $\mathrm{C}$ - и $\mathrm{Si}-г$ ранях политипов $3 C$-, $6 H$ - и $4 H$-SiC рассмотрена в рамках модели Халдейна-Андерсона. Переход заряда с адатома бария на подложку проанализирован с учетом зонных и локальных состояний адсорбционной системы. Оценены ионный и металлический вклады в энергию адсорбции. Для проверки некоторых результатов использована модель поверхностной молекулы.

Ключевые слова: зонные и локальные состояния, переход заряда, металлическая и ионная составляющие энергии адсорбции.

DOI: 10.21883/PJTF.2020.01.48857.18050

Многочисленные политипы карбида кремния, являющиеся широкозонными полупроводниками и характеризуемые высокой стойкостью к температурным, механическим и радиационным воздействиям, представляют большой интерес для создания различных приборных структур [1-4], а в последнее время используются и как подложки для получения слоев графена [5]. Несмотря на указанную популярность карбида кремния, его адсорбционные свойства изучены весьма слабо. В работах [6,7] мы приступили к модельному описанию адсорбции атомов и молекул на $\mathrm{SiC}$ при низких степенях покрытия, когда адчастицу можно рассматривать как изолированную. В настоящей работе мы рассмотрим адсорбцию атомов бария на $\mathrm{C}$ - и $\mathrm{Si}$-гранях политипов карбида кремния. Интерес именно к этой адсорбционной системе не в последнюю очередь вызван результатами работы [8], где экспериментально исследовалась гетероструктура $\mathrm{Ba} / \mathrm{SiC} / \mathrm{Si}(111)-8^{\circ}$ и было продемонстрировано возникновение новой углеродной наноструктуры.

Модельный подход к проблеме адсорбции изложен в $[9,10]$. Необходимо отметить, что свободный атом бария содержит на внешней $s$-оболочке два электрона, между которыми действует внутриатомное кулоновское отталкивание. Поскольку на переход заряда между адатомом и подложкой „работает“ только один электрон, можно свести задачу к одноэлектронной (по аналогии с работой [11], где рассматривалась адсорбция ванадия на рутиле). Тогда плотность состояния на адатоме имеет вид

$$
\rho_{a}(\omega)=\frac{1}{\pi} \frac{\Gamma(\omega)}{\left[\omega-\varepsilon_{a}-\Lambda(\omega)\right]^{2}+\Gamma^{2}(\omega)},
$$

Здесь $\omega-$ энергетическая переменная; $\varepsilon_{a}-$ энергия одноэлектронного уровня адчастицы; $\Gamma(\omega)=\pi V^{2} \rho_{s u b}(\omega)-$ функция уширения квазиуровня адчастицы, где $\rho_{\text {sub }}(\omega)$ - плотность состояний субстрата, $V$ - матричный элемент взаимодействия адчастица-подложка;
$\Lambda(\omega)=V^{2} \int_{-\infty}^{\infty} \rho_{s u b}\left(\omega^{\prime}\right)\left(\omega-\omega^{\prime}\right)^{-1} d \omega^{\prime}-$ функция сдвига квазиуровня. Для описания плотности состояний полупроводниковой подложки весьма удобна простая модель Халдейна-Андерсона $[6,7,9,10]$, в которой $\rho_{\text {sub }}(\omega)=\rho_{s}$ для $\left|\omega-E_{0}\right| \geqslant E_{g} / 2$ и $\rho_{\text {sub }}(\omega)=0$ для $\left|\omega-E_{0}\right|<E_{g} / 2$, где $E_{0}=\chi+E_{g} / 2-$ энергия центра запрещенной зоны относительно вакуума, принимаемая за начало отсчета, $\chi$ - электронное сродство политипа карбида кремния [12]. Тогда $\Gamma \equiv \pi V^{2} \rho_{s}=$ const и $\Lambda(\omega)=(\Gamma / \pi) \ln \left|\left(\omega-E_{g} / 2\right) /\left(\omega+E_{g} / 2\right)\right|$.

Энергия квазиуровня адатома относительно центра запрещенной зоны карбида кремния равна $\varepsilon_{a}=-I+e^{2} / 4 d+E_{0}$, где $I=5.21 \mathrm{eV}[13]$ - энергия ионизации атома $\mathrm{Ba} ; d$ - длина адсорбционной связи; $e$ - заряд электрона $[6,7,9,10]$. Для адсорбции на С-грани $d=r_{a}(\mathrm{Ba})+r_{a}(\mathrm{C})=3.01 \AA$, так как атомные радиусы бария и углерода равны соответственно 2.24 и $0.77 \AA$ [13]; для адсорбции на $\mathrm{Si}$-грани $d=r_{a}(\mathrm{Ba})+$ $+r_{a}(\mathrm{Si})=3.42 \AA$, так как $r_{a}(\mathrm{Si})=1.18 \AA[13]$. Энергетические характеристики для $3 \mathrm{C}$-, $6 H$ - и $4 H$-политипов $\mathrm{SiC}$ сведены в табл. 1, там же приведены значения $\varepsilon_{a}$.

Число заполнения адчастицы $n_{a}$ равно сумме зонного $n_{v}$ и локального $n_{l}$ вкладов $[6,7,9,10]$. Как показано в [9], вклад валентной зоны $n_{v}$ может быть вычислен по следующей приближенной формуле:

$$
n_{v}=\frac{1}{\pi} \operatorname{arcctg} \frac{\varepsilon_{a}+R}{\Gamma}, \quad R=\frac{E_{g}}{2} \sqrt{1+\frac{4 \Gamma}{\pi E_{g}}} .
$$

Полуширина квазиуровня адатома $\Gamma$ рассматривается обычно как подгоночный параметр. При $\Gamma=E_{g} / 8$ получаем значения $n_{v}$, приведенные в табл. 1. Далее проанализируем, как величина Г влияет на результаты.

Энергия локального состояния $\omega_{l}$, где $\left|\omega_{l}\right|<E_{g} / 2$, находится из решения уравнения $\omega-\varepsilon_{a}-\Lambda(\omega)=0$, а 
Таблица 1. Характеристики системы $\mathrm{Ba} / \mathrm{SiC}$ : ширина запрещенной зоны $E_{g}$, сродство к электрону $\chi$, энергия квазиуровня адатома бария относительно центра запрещенной зоны $\varepsilon_{a}$, зонный вклад в числа заполнения $n_{v}$ (все энергетические величины приведены в $\mathrm{eV}$ )

\begin{tabular}{c|c|c|c}
\hline \multirow{2}{*}{ Параметр } & \multicolumn{3}{|c}{ Политип } \\
\cline { 2 - 4 } & $3 C$ & $6 H$ & $4 H$ \\
\hline$E_{g}$ & 2.40 & 3.00 & 3.23 \\
$\chi$ & 4.00 & 3.45 & 3.17 \\
$E_{0}$ & 5.20 & 4.95 & 4.785 \\
\multicolumn{4}{|c|}{ С-грань } \\
$\varepsilon_{a}$ & $1.19 \quad 0.94$ & 0.77 \\
$n_{v}$ & 0.04 & 0.05 & 0.05 \\
\multicolumn{2}{|c|}{ Si-грань } \\
$\varepsilon_{a}$ & 1.04 & 0.79 & 0.63 \\
$n_{v}$ & 0.04 & 0.05 & 0.05 \\
\end{tabular}

Таблица 2. Энергия локального уровня $\omega_{l}$ и число его заполнения $\bar{n}_{l}$ при $\omega_{l}<E_{\mathrm{F}}$, ионная составляющая $E_{a d s}^{\text {ion }}$ и полная энергия адсорбции $E_{a d s}$ для системы $\mathrm{Ba} / \mathrm{SiC}\left(E_{a d s}^{i o n}>\right.$ соответствует случаю $\omega_{l}>E_{\mathrm{F}}, E_{a d s}^{i o n}<-$ случаю $\omega_{l}<E_{\mathrm{F}}$, все энергетические величины приведены в $\mathrm{eV}$ )

\begin{tabular}{|c|c|c|c|}
\hline \multirow{2}{*}{ Параметр } & \multicolumn{3}{|c|}{ Политип } \\
\hline & $3 C$ & $6 H$ & $4 H$ \\
\hline$\omega_{l}$ & & & \\
\hline & 0.97 & 0.82 & 0.68 \\
\hline $\begin{array}{c}\mathrm{Si}-г р а н ь ~ \\
\bar{n}_{l}\end{array}$ & 0.86 & 0.69 & 0.56 \\
\hline С-грань & 0.69 & 0.83 & 0.81 \\
\hline Si-грань & 0.75 & 0.85 & 0.85 \\
\hline \multicolumn{4}{|c|}{ С-грань } \\
\hline$-E_{a d s}^{i o n}>$ & 0.09 & 0.02 & 0.02 \\
\hline$-E_{a d s}^{i o n}<$ & 1.10 & 1.08 & 1.08 \\
\hline$-E_{a d s}>$ & 0.28 & 0.21 & 0.21 \\
\hline$-E_{a d s}<$ & 1.29 & 1.27 & 1.27 \\
\hline \multicolumn{4}{|c|}{ Si-грань } \\
\hline$-E_{a d s}^{i o n}>$ & 0.09 & 0.02 & 0.02 \\
\hline$-E_{a d s}^{i o n}<$ & 0.97 & 0.95 & 0.95 \\
\hline$-E_{a d s}>$ & 0.28 & 0.21 & 0.21 \\
\hline$-E_{a d s}<$ & 1.16 & 1.14 & 1.14 \\
\hline
\end{tabular}

число заполнения этого состояния равно

$$
n_{l}=\bar{n}_{l} \Theta\left(E_{\mathrm{F}}-\omega_{l}\right), \bar{n}_{l}=\left(1+\frac{\Gamma}{\pi} \frac{E_{g}}{\left(E_{g} / 2\right)^{2}-\omega_{l}^{2}}\right)^{-1},
$$

где функция Хэвисайда $\Theta\left(E_{\mathrm{F}}-\omega_{l}\right)$ гарантирует, что учитываются вклады только от тех уровней, которые лежат ниже уровня Ферми $E_{\mathrm{F}}[9,10]$. Значения $\bar{n}_{l}$ приведены в табл. 2.

Энергию адсорбции $E_{a d s}$ можно представить в виде суммы ионной $E_{a d s}^{i o n}$ и металлической $E_{a d s}^{m e t}$ состав- ляющих $[9,10]$, первую из которых можно оценить по формуле

$$
E_{a d s}^{i o n}=-\frac{(Z e)^{2}}{4 d}
$$

где $Z=(1-n)$ - заряд адатома (или переход заряда между адатомом и подложкой). В табл. 2 приведены значения $E_{a d s}^{i o n}$ для случаев, когда локальные уровни лежат выше фермиевского уровня и поэтому пусты $\left(E_{a d s}^{i o n}>\right)$ и когда эти уровни находятся под уровнем Ферми и поэтому заполнены $\left(E_{a d s}^{i o n}<\right)$.

Для оценки металлической составляющей энергии адсорбции $E_{a d s}^{m e t}$ воспользуемся соотношением неопределенностей Гейзенберга $\Delta x \Delta p \sim \hbar$. Полагая, что в изолированном атоме $\Delta x \sim r_{a}(\mathrm{Ba})$, а в адсорбированном состоянии $\Delta x \sim d$, получим выигрыш в кинетической энергии $\Delta E_{k i n} \sim\left(\hbar^{2} / 2 m d^{2}\right)\left[1-\left(r_{a} / d\right)^{2}\right]$, где $m-$ масса свободного электрона. Как и в [9], принимаем эту величину за простейшую порядковую оценку металлической составляющей энергии адсорбции

$$
E_{a d s}^{m e t}=-\frac{\hbar^{2}}{2 m d^{2}}\left(1-\frac{r_{a}^{2}}{d^{2}}\right) .
$$

Для обеих граней получаем $E_{a d s}^{m e t}=-0.19 \mathrm{eV}$. Теперь легко найти значения суммарной энергии адсорбции $E_{a d s}$ (табл. 2).

Проанализируем полученные результаты. В ряду политипов $3 \mathrm{C} \rightarrow 6 \mathrm{H} \rightarrow 4 \mathrm{H}$ уровень $\varepsilon_{a}$ сдвигается к центру запрещенной зоны политипа, оставаясь при этом в верхней ее половине. Такой сдвиг уровня $\varepsilon_{a}$ ведет к увеличению зонного вклада $n_{v}$ в число заполнения $n$. В том же ряду локальные уровни $\omega_{l}$ и числа их заполнения $\bar{n}_{l}$ ведут себя аналогично $\varepsilon_{a}$ и $n_{v}$. Разница, однако, состоит в том, что $n_{v}<<1$, тогда как значение $\bar{n}_{l}$ близко к единице. Если уровень Ферми лежит вблизи потолка валентной зоны $(p-\mathrm{SiC})$, заряд адатома бария $Z$ близок к + 1. Когда же уровень Ферми расположен вблизи дна зоны проводимости $(n-\mathrm{SiC})$, имеем $Z \sim 0$. Отсюда следует, что в первом случае ионная составляющая энергии адсорбции $E_{a d s}^{i o n} \sim 1 \mathrm{eV}$, что приблизительно в 5 раз превосходит по модулю металлическую составляющую $E_{a d s}^{m e t}$; во втором случае $E_{a d s}^{i o n} \sim 0$ и $E_{a d s} \approx E_{a d s}^{m e t}$. На рисунке представлены зависимости рассчитанных величин от полуширины квазиуровня. Из рисунка следует, что вклады в числа заполнения $n_{v}$ и $\bar{n}_{l}$ монотонно и достаточно слабо зависят от $\Gamma(a)$. То же относится и к энергиям $\omega_{l}$ и $E_{a d s}^{i o n}$. Поэтому сравнительно произвольное задание $\Gamma$ качественно не меняет полученных результатов.

Для альтернативной оценки перехода заряда и энергии связи атома бария с подложкой воспользуемся моделью поверхностной двухатомной молекулы $(\mathrm{BaC}, \mathrm{BaSi})$ и методом связывающих орбиталей Харрисона [14,15]. Энергии $s p^{3}$-орбиталей $\varepsilon_{h}=\left(\varepsilon_{s}+3 \varepsilon_{p}\right) / 4$ (где $\varepsilon_{s}$ и $\varepsilon_{p}$ - энергии $s$ - и $p$-состояний) атомов $\mathrm{C}$ и $\mathrm{Si}$ равны соответственно $\varepsilon_{h}(\mathrm{C})=-11.11 \mathrm{eV}$ и $\varepsilon_{h}(\mathrm{Si})=-8.28 \mathrm{eV}$, так как $\quad \varepsilon_{s}(\mathrm{C})=-17.52 \mathrm{eV}, \quad \varepsilon_{p}(\mathrm{C})=-8.97 \mathrm{eV}$, $\varepsilon_{s}(\mathrm{Si})=-13.55 \mathrm{eV}, \varepsilon_{p}(\mathrm{Si})=-6.52 \mathrm{eV}$ (таблицы атомных 

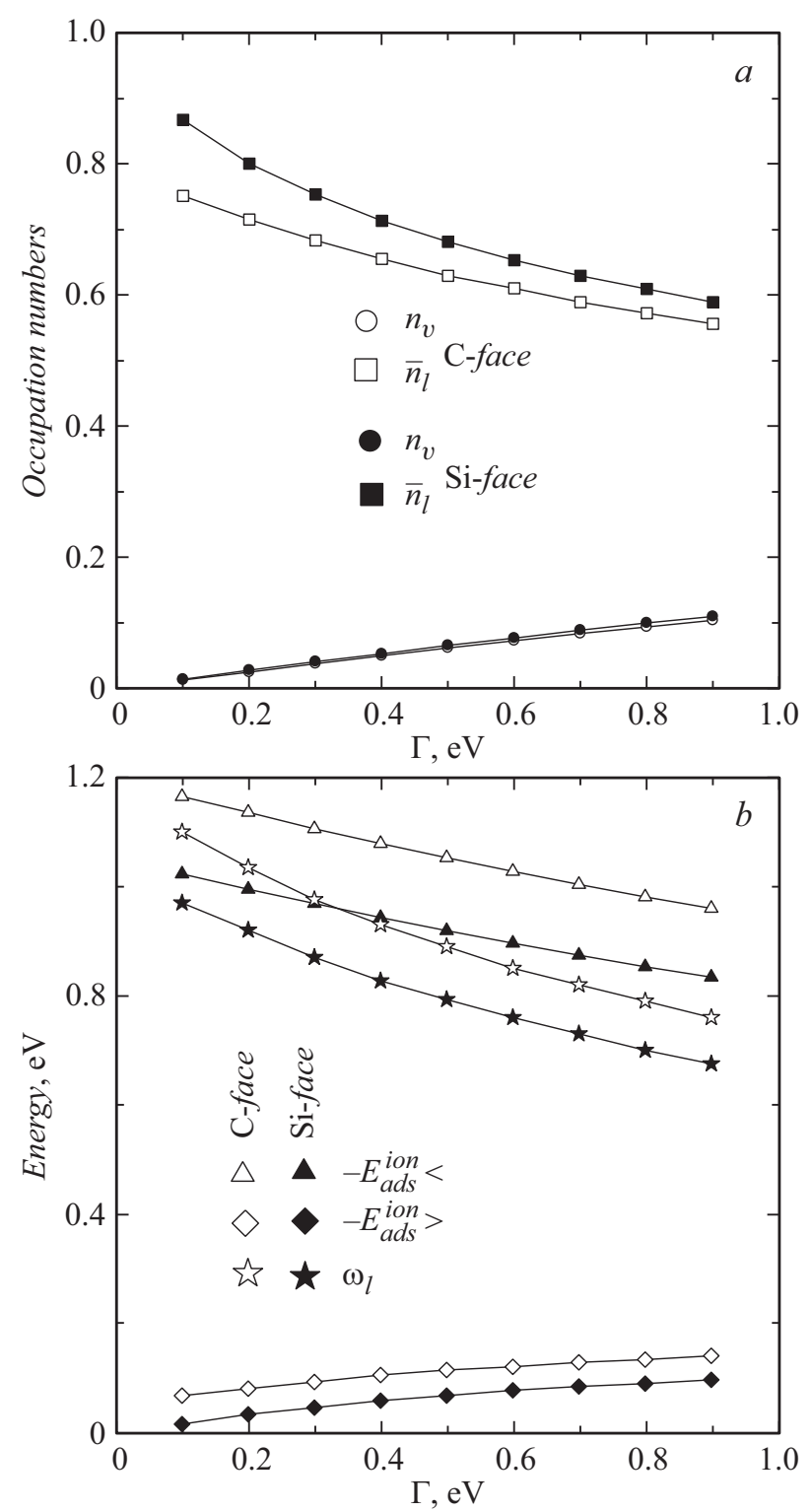

Адсорбция атома бария на C- и $\mathrm{Si}-г р а н я х ~ 3 C$-политипа карбида кремния. $a-$ зависимости зонных $n_{v}$ (кружки) и локальных $\bar{n}_{l}$ (квадраты) вкладов в суммарное число заполнения от полуширины квазиуровня адатома $\Gamma ; b-$ зависимости взятых с обратным знаком ионных вкладов $-E_{a d s}^{i o n}<$ (треугольники) и $-E_{a d s}^{i o n}>$ (ромбы, значения увеличены в 10 раз) и энергии локального уровня $\omega_{l}$ (звездочки) от полуширины квазиуровня адатома Г. Светлые символы соответствуют адсорбции на C-грани, темные - адсорбции на $\mathrm{Si}$-грани; $E_{a d s}^{i o n}>$ соответствует случаю $\omega_{l}>E_{\mathrm{F}}, E_{a d s}^{i o n}<-$ случаю $\omega_{l}<E_{\mathrm{F}}$. Энергетический интервал на части $b$ совпадает с верхней половиной запрещенной зоны $3 C$-SiC.

термов Хермана-Скиллмана [14]). Рассмотрим $\sigma$-связь $s$-орбитали атома Ва с $s p^{3}$-орбиталью атомов С и $\mathrm{Si}$. Поскольку $\varepsilon_{s}(\mathrm{Ba})=-4.45 \mathrm{eV}$ [14], получим полярные энергии

$$
\begin{aligned}
& V_{3}(\mathrm{Ba}-\mathrm{C})=\left(\varepsilon_{h}(\mathrm{C})-\varepsilon_{s}(\mathrm{Ba})\right) / 2=3.33 \mathrm{eV}, \\
& V_{3}(\mathrm{Ba}-\mathrm{Si})=\left(\varepsilon_{h}(\mathrm{Si})-\varepsilon_{s}(\mathrm{Ba})\right) / 2=1.92 \mathrm{eV} .
\end{aligned}
$$

Ковалентная энергия связи равна $V_{2}=\left[\left(\eta_{s s \sigma}+\eta_{s p \sigma} \sqrt{3}\right) / 2\right]\left(\eta^{2} / m d^{2}\right) \quad[16], \quad$ где $m-$ масса электрона, $\hbar_{s s \sigma}=1.32, \hbar_{s p \sigma}=1.42$ [15], так что $V_{2}(\mathrm{Ba}-\mathrm{C})=1.59 \mathrm{eV}$ и $V_{2}(\mathrm{Ba}-\mathrm{Si})=1.23 \mathrm{eV}$. Полярность связи определяется как $\alpha_{p}=V_{3} / \sqrt{V_{2}^{2}+V_{3}^{2}}$, откуда $\alpha_{c}(\mathrm{Ba}-\mathrm{C})=0.90 \quad$ и $\quad \alpha_{c}(\mathrm{Ba}-\mathrm{Si})=0.84$. Переходя $\quad$ к задаче об адсорбции и полагая $Z=\alpha_{p}$, из (4) получим $E_{\text {ads }}^{i o n}(\mathrm{Ba}-\mathrm{C})=-0.97 \mathrm{eV}$ и $E_{a d s}^{i o n}(\mathrm{Ba}-\mathrm{Si})=-0.74 \mathrm{eV}$, что достаточно хорошо согласуется со значениями $E_{a d s}^{i o n}<$, приведенными в табл. 2. Таким образом, в рамках двух различных подходов мы установили, что в случае $p$-SiC связь адатома бария с подложкой носит ярко выраженный ионный характер.

К сожалению, мы не имеем возможности сопоставить полученные нами результаты с экспериментальными данными по энергии адсорбции $\mathrm{Ba}$ на $\mathrm{SiC}$. В отличие от адсорбции металлов на металлах [17] адсорбция металлов на полупроводниках изучена слабо, так что авторам не удалось найти данных даже по энергии адсорбции бария на кремнии и алмазе, хотя некоторые данные по этим (или близким) адсорбционным системам имеются (см. $[18,19]$ и $[20,21])$. Обратимся поэтому для сравнения к адсорбции цезия. По результатам расчета из первых принципов для системы $\mathrm{Cs} / \mathrm{Si}(001)$ при степени покрытия $\Theta=0.5$ значения энергии адсорбции для различных адсорбционных позиций лежат в интервале -(1.46-2.46) eV [22]. Аналогичные расчеты для атомов цезия на графите дают энергию адсорбции, равную $-1.42 \mathrm{eV}$ [23]. В настоящее время приходится ограничиться лишь такой условной проверкой полученных нами результатов.

\section{Благодарности}

Авторы признательны С.А. Кукушкину за предложение темы и полезные обсуждения.

\section{Конфликт интересов}

Авторы заявляют, что у них нет конфликта интересов.

\section{Список литературы}

[1] Silicon carbide: recent major advances / Eds W.J. Choyke, H. Matsunami, G. Pensl. Berlin-Heidelberg: Springer, 2004. $899 \mathrm{p}$.

[2] Advances in silicon carbide. Processing and applications / Eds S.E. Saddow, A. Agarwal. Boston-London: Artech House, 2004. $228 \mathrm{p}$.

[3] Lebedev A.A. // Semicond. Sci. Technol. 2006. V. 21. N 6. P. R17-R34.

[4] Liu G., Tuttle B.R., Dhar S. // Appl. Phys. Rev. 2015. V. 2. N 2. P. 021307.

[5] Wu Y.H., Yu T., Chen Z.X. // J. Appl. Phys. 2010. V. 108. N 7. P. 071301.

[6] Давыдов С.Ю., Посредник О.В. // ФТТ. 2019. Т. 61. В. 8. C. 1538-1541. 
[7] Давыдов С.Ю., Посредник О.В. // ФТТ. 2020. Т. 62. В. 2. C. 298-301.

[8] Бенеманская Г.В., Дементьев П.А., Кукушкин С.А., Осипов А.В., Тимошнев С.Н. // Письма в ЖТФ. 2019. Т. 45. B. 5. C. $17-20$.

[9] Давыдов С.Ю., Трошин С.В. // ФТТ. 2007. Т. 49. В. 8. C. $1508-1513$.

[10] Давыдов С.Ю., Лебедев А.А., Посредник О.В. Элементарное введение в теорию наносистем. СПб.: Лань, 2014. $191 \mathrm{c}$.

[11] Давыдов С.Ю., Павлык А.В. // Письма в ЖТФ. 2003. Т. 29. B. 12. C. $33-36$.

[12] Давыдов С.Ю. // ФТП. 2007. Т. 41. В. 6. С. 718-720.

[13] Физические величины. Справочник / Под ред. Е.С. Григорьева, Е.3. Мейлихова. М.: Энергоатомиздат, 1991. $1232 \mathrm{c}$.

[14] Харрисон У. Электронная структура и свойства твердых тел. М.: Мир, 1983. 381 с.

[15] Harrison W.A. // Phys. Rev. B. 1983. V. 27. N 6. P. $3592-$ 3604.

[16] Давыдов С.Ю., Тихонов С.К. // ФТТ. 1995. Т. 37. В. 9. C. 2749-2754.

[17] Браун О.М., Медведев В.К. // УФН. 1989. Т. 157. В. 4. C. 631-666.

[18] Takeda Y., Urano T., Ohtani T., Tamiya K., Hongo S. // Surf. Sci. 1998. V. 402-404. P. 692-696.

[19] Yao X., Hu X., Sarid D., Yu Z., Wang J., Marshall D.S., Droopad R., Abrokwah J.K., Hallmark J.A., Ooms W.J. // Phys. Rev. B. 1999. V. 59. N 7. P. 5115-5119.

[20] O'Donnell K.M., Martin T.L., Allan N.L. // Chem. Mater. 2015. V. 27. N 4. P. 1306-1315.

[21] O'Donnell K.M., Edmonds M.T., Tadich A., Thomsen L., Stacey A., Schenk A., Pakes C.I., Ley L. arXiv: 1505.06410.

[22] Shaltaf R., Mete E., Ellialtoğlu Ş. // Phys. Rev. B. 2005. V. 72. N 20. P. 205415.

[23] Luo X., Fang C., Li X., Lai W., Liang T. // J. Nucl. Mater. 2013. V. 441. N 1-3. P. 113-118. 